\title{
An open-label, randomized, placebo-controlled study on the effectiveness of a novel probiotics administration protocol (ProbiotiCKD) in patients with mild renal insufficiency (stage 3a of CKD)
}

\author{
Mariadelina Simeoni ${ }^{1} \cdot$ Maria Lucia Citraro ${ }^{1}$. Annamaria Cerantonio ${ }^{1} \cdot$ Francesca Deodato $^{1} \cdot$ Michele Provenzano $^{1}$. \\ Paola Cianfrone ${ }^{1}$ - Maria Capria ${ }^{1}$. Silvia Corrado ${ }^{1}$ - Emanuela Libri ${ }^{1}$ - Alessandro Comi ${ }^{1}$ - Arturo Pujia ${ }^{2}$. \\ Ludovico Abenavoli ${ }^{3} \cdot$ Michele Andreucci $^{1} \cdot$ Massimo Cocchi $^{4} \cdot$ Tiziana Montalcini $^{2} \cdot$ Giorgio Fuiano $^{1}$
}

Received: 8 December 2017 / Accepted: 17 July 2018 / Published online: 3 August 2018

(c) The Author(s) 2018, corrected publication October 2018

\begin{abstract}
Purpose Gut dysbiosis has been described in advanced, but not in initial stages of CKD. Considering the relevant impact of gut dysbiosis on renal and cardiovascular risk, its diagnosis and treatment are clinically relevant.

Methods We designed, open-label, placebo-controlled intervention study (ProbiotiCKD) to evaluate gut microbiota metabolism in a cohort of KDIGO CKD patients $(n=28)$ at baseline and after a randomly assigned treatment with probiotics or placebo. Gut microbiota status was evaluated on:.

Results Basal mean fecal Lactobacillales and Bifidobacteria concentrations were abnormally low in both groups, while urinary indican and 3-MI levels were, indicating a mixed (fermentative and putrefactive) dysbiosis. After treatment, mean fecal Lactobacillales and Bifidobacteria concentrations were increased, only in the probiotics group $(p<0.001)$. Conversely, mean urinary indican and 3-MI levels only in the group treated with probiotics $(p<0.001)$. Compared to placebo group, significant improvements of C-reactive protein $(p<0.001)$, iron $(p<0.001)$, ferritin $(p<0.001)$, transferrin saturation $(p<0.001)$, $\beta 2$-microglobulin $(p<0.001)$, serum iPTH and serum calcium were observed only in the probiotics group.

Conclusions ProbiotiCKD is the first intervention study demonstrating that an intestinal mixed dysbiosis is present even in early CKD stage and can be effectively corrected by the novel mode of administration of high-quality probiotics with improvement of inflammatory indices, iron status and iPTH stabilization.
\end{abstract}

Keywords Chronic kidney disease $\cdot$ Uremia $\cdot$ Gut microbiota $\cdot$ Dysbiosis $\cdot$ Probiotics

\section{Introduction}

Mariadelina Simeoni

adelina.simeoni@unicz.it; adelina_simeoni@yahoo.it

1 Nephrology Unit, Department of Surgical and Medical Science, 'Magna Graecia' University Hospital, Viale Europa, Germaneto Area, 88100 Catanzaro, CZ, Italy

2 Clinical Nutrition Unit, 'Magna Graecia' University Hospital, 88100 Catanzaro, CZ, Italy

3 Digestive Physiopathology Unit, 'Magna Graecia' University Hospital, 88100 Catanzaro, CZ, Italy

4 "Paolo Sotgiu" Institute for Research in Quantitative and Quantum Psychiatry and Cardiology, LUdeS, Lugano, Switzerland
The human intestinal microbiota is a vast pool of symbiotic microorganisms living in the human gut and it is involved in important metabolic, trophic and immunological functions in the host [1]. 95\% of microbiota is composed of anaerobic bacteria and 5\% of aerobic bacteria [2]. Bacteria concentrations vary along the gut with an exponential increase in the fecal direction [3]. As for recent reports, fecal concentrations of Lactobacillales and Bifidobacteria range $1 \times 10^{7}$ to $1 \times 10^{10} \mathrm{CFU} / \mathrm{g}$ [4] and $1 \times 10^{8}$ to $1 \times 10^{10} \mathrm{CFU} / \mathrm{g}$ [5], respectively.

Gut dysbiosis consists of a significant alteration in microbiota composition and it is prevalently characterized by a concentration of Lactobacillales and Bifidobacteria 
and a prevalence of aerobic bacteria. Gut dysbiosis is associated with both intestinal and extra-intestinal reflexes. Of note, intestinal dysbiosis promotes atherosclerosis and hypertension and is involved in the activation of several molecular pathways of cardiovascular risk worsening [6].

Chronic kidney disease (CKD), a relevant multifactorial [7-9] health problem associated with poor quality of life [10], high management costs and increased death risk. In several studies conducted on both animals and humans, intestinal dysbiosis has been found in or end-stage renal disease (ESRD). Microbiota disequilibrium in ESRD patients depends on uremic and non-uremic factors, the latter based on dietary and pharmacological approach to CKD patients [11]. In advanced CKD stage vegetables and fruit intake has to be restricted to prevent the risk of hyperkalemia and fluid overload. This fiber shortage amplifies the predisposing factors to dysbiosis, such as intestinal transit slowing, intestinal wall edema and metabolic acidosis increase [12, 13]. Furthermore, ESRD imposes an increase of oral drugs (iron and vitamin D analogs supplementation, potassium and phosphate-chelating agents, diuretics) intake inducing pro-inflammatory gastrointestinal overload $[14,15]$. Consequently, intestinal flora metabolism is greatly modified in uremic patients with prevalence of proteolytic and/or saccharolytic fermentation process with increased production and reabsorption of intestinal bacterial metabolites, such as indican and 3-methyl-indole (3-MI) [16, 17].

3-methyl-indole and indican are currently used to diagnose either a and/or a fermentative intestinal dysbiosis, respectively [18-23]. Fermentative dysbiosis is due to nonabsorbed sugar hydrolization by several bacteria strains in the ascendant colon and in the caecum. Acetic acid, water and carbonic anhydride are produced by fermentation and cause intestinal wall edema, diarrhea, odorless meteorism and functional alteration of gut-associated lymphoid tissue (GALT) and mucosa-associated lymphoid tissue (MALT) [24]. Putrefactive dysbiosis, as previously described, is triggered by an overgrowth of intestinal putrefactive microorganisms favored by fecal $\mathrm{pH}$ changes and an unbalanced dietary intake of fibers, proteins and fats. The main symptoms of putrefactive dysbiosis are constipation, smelly meteorism, digestive difficulties, impaired GALT and MALT functions [25].

Moreover, it has been widely reported that metabolites of altered gut microbiota are directly involved in mechanisms of cardiovascular disease and renal damage progression [26-29].

We present the results of ProbiotiCKD study. This investigation was addressed to characterize gut microbiota status and metabolism in a cohort of patients with 3a stage CKD. ProbiotiCKD clinical trial tested the impact of a novel optimized probiotics administration protocol on urinary indican and 3-MI levels, all fecal Lactobacillales and Bifidobacteria concentrations, and serum biochemistry lab parameters.

\section{Materials and methods}

We designed ProbiotiCKD protocol to be tested in a singlecenter, open-label, placebo-controlled intervention study. Patients with stable CKD stage $3 \mathrm{a}$, referring to the Nephrology Unit at the University Hospital of Catanzaro (Italy), between January 2016 and March 2017, were progressively screened, as reported in Fig. 1. The eligible patients were studied to evaluate the impact on intestinal microbiota of a novel probiotics administration protocol vs placebo.

To be enrolled, patients had to meet the following criteria: age $>18$ years; Caucasian race; Epidemiology Collaboration Equation (EPI) estimated-GFR (eGFR) ranging $60-45 \mathrm{ml} /$ $\mathrm{min} / 1.73 \mathrm{~m}^{2}$. Conversely, patients meeting Montreal classification criteria for inflammatory bowel diseases [30] were excluded. In addition, patients with malabsorption, autoimmune systemic diseases or cancer were excluded, as well as kidney transplant recipients, pregnant women and patients on current or recent antibiotic therapy or immunosuppressant drugs. To standardize baseline conditions, any prebiotic or probiotic supplement had to be suspended at least 1 month before enrollment. However, according to current nutritional recommendations to CKD patients [31,32] and to obtain a satisfactory dietary prebiotic intake, the enrolled subjects had to adhere to a protein dietary intake ranging $0.7-1 \mathrm{~g} /$ $\mathrm{kg} /$ day, also assuring a daily consumption of two pieces of fruit (apple or pear) and $200 \mathrm{~g}$ of double-boiled leafy green vegetables (the double boiling was used to discharge vegetables' potassium content) [33]. Patients were provided with a weekly dietary diary to be filled in. The diaries had to be analyzed by our nutritionist, to make sure that background dietary conditions were adequate and uniform.

Eligible patients agreeing protocol requirements, had to provide written informed consent to participate in the ProbiotiCKD study, which was conducted in accordance with the Helsinki Declaration.

At baseline, demographic data, medical history, dietary diaries and concomitant therapy were collected. A careful physical examination was also performed. In addition, a fasting peripheral venous blood sample was drawn for serum biochemistry and plasma intact parathormone (iPTH) assessment. Basal eGFR was calculated by CKD-EPI equation. In addition, a cultural quantitative analysis of two different fecal bacteria clusters (Lactobacillales and Bifidobacteria) were rapidly analyzed by anaerobic technique at the Microbiology Unit of University Hospital of Catanzaro. According to previous studies, any stool sample in which Lactobacillales and Bifidobacteria concentrations were found $<1 \times 10^{7} \mathrm{CFU} / \mathrm{g}$ and $<1 \times 10^{8} \mathrm{CFU} / \mathrm{g}$ respectively, 


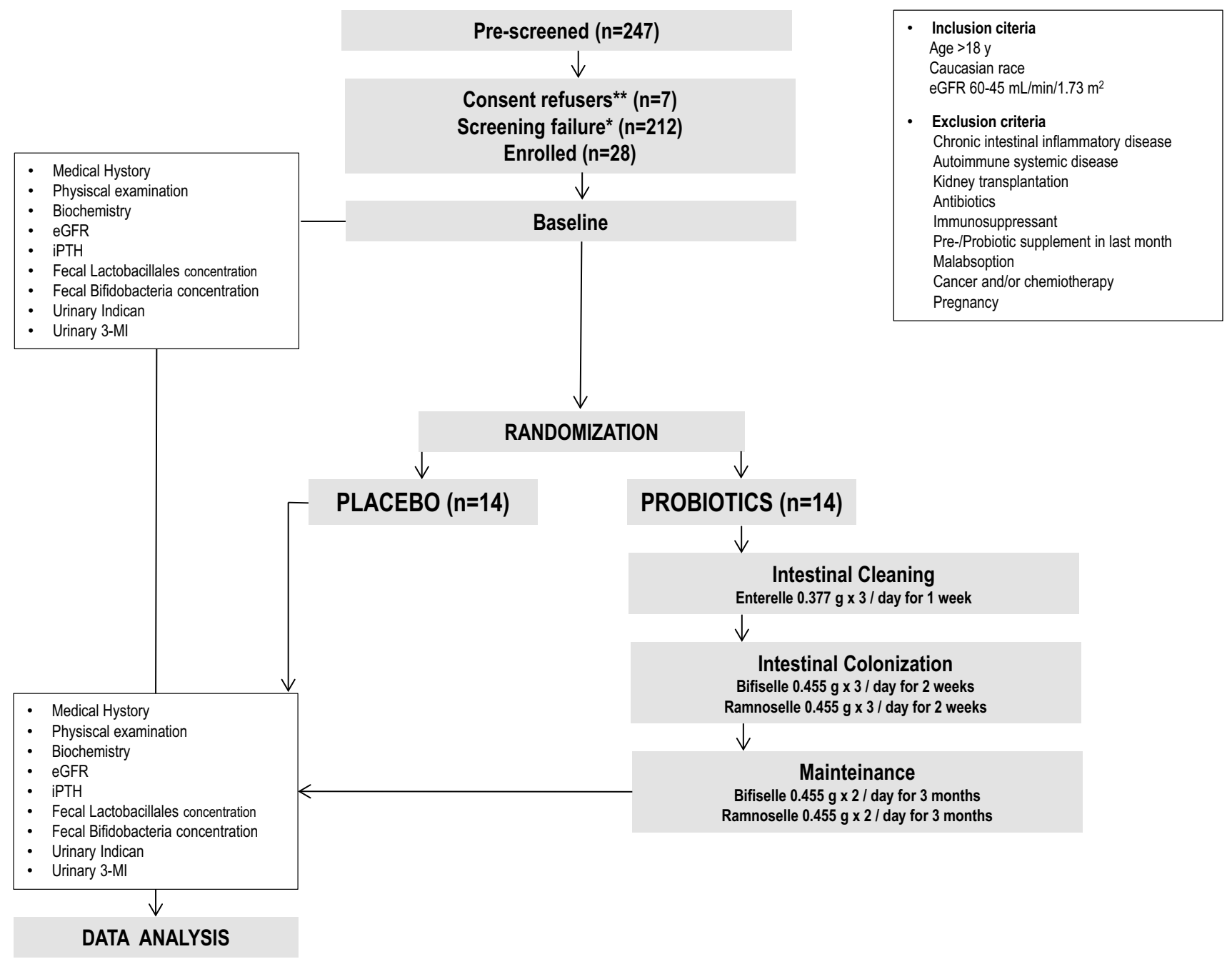

Fig. 1 Study design *excluded after screening; **did not release the informed consent

was considered associated to an altered gut microbiota [4, $5,22]$. All patients had to collect a morning spot urine sample for 3-MI and indican assessment by high-performance liquid chromatography and colorimetric technique, respectively. As for the 3-MI determination, aliquots of $300 \mu \mathrm{l}$ of acetonitrile were added to an equal volume of each urine sample. The obtained solutions were vortexed for $3 \times 5 \mathrm{~s}$ and chilled at $4{ }^{\circ} \mathrm{C}$ for $15 \mathrm{~min}$. Afterwards, samples were centrifuged for $5 \mathrm{~min}$ at $12,000 \mathrm{rpm}\left(1^{\circ} \mathrm{C}\right)$. Clear supernatants were then used to perform chromatography analysis on an Agilent 1100 LC-MS system. Chromatographic separation was obtained on a Waters XBridge column $(3.5 \mu \mathrm{m}$, $4.6 \times 150 \mathrm{~mm}$; Waters, Milford, MA, USA), operating at $40{ }^{\circ} \mathrm{C}[34]$.

For urinary indican determination, a standard colorimetric assay kit was used according to the procedures indicated by the manufacturer (https://www.sigmaaldrich.com/ content/dam/sigma-aldrich/docs/Sigma/Bulletin/2/mak12 8bul.pdf). According to our laboratory reference ranges and to previous observations [23, 35-39], urinary indican and 3-MI were considered normal for values lower than 10 and $10 \mu \mathrm{g} / \mathrm{l}$, respectively. Creatininuria was also measured to calculate the urine indican and 3-MI to creatinine ratios.

Patients were randomized by a computer-generated random number list to receive either placebo or probiotics. Probiotics are defined by the United Nations Food and Agriculture Organization and the World Health Organization as "live microorganisms" that, when administered in adequate amounts, confer health benefits to the host [40, 41]. The use of different complexes of high concentration and stable probiotics in a 'sequential' manner was the novel administration mode tested in ProbiotiCKD study. The probiotics administration protocol was designed by nephrologists, nutritionists and gastroenterologists at our University Hospital, after an accurate review of the current literature. 
In particular, the treated group underwent the following three phases of the treatment including the 'sequential' use of different probiotics with: (a) phase 1: intestinal cleaning by oral administration during main meals (breakfast, lunch and dinner) for 1 week of one capsule of a complex of probiotics (Enterelle $0.377 \mathrm{~g}{ }^{\circledR}$ Bromatech) composed of Enterococcus faecium (UBEF-41), Lactobacillus acidophilus (LA-14) and Saccharomyces cerevisiae subspecies Boulardii (MTCC-5375); (b) phase 2: intestinal colonization with oral administration, for 2 weeks, during breakfast, lunch and dinner of one capsule of a complex of Bifidobacteria (Bifiselle ${ }^{\circledR B}$ Bromatech $0.455 \mathrm{~g}$ ) composed of Bifidobacterium brevis (BB03), Bifidobacterium bifidum (BB06), Bifidobacterium longum (BL05) and one capsule of another probiotics complex (Ramnoselle ${ }^{\circledR}$ Bromatech $0.455 \mathrm{~g}$ ) composed of Lactobacillus rhamnosus (HN-001), Lactobacillus rhamnosus (LR-32) and Lactobacillus acidophilus (LA14); (c) phase 3: microbiota maintenance by both Bisifelle and Ramnoselle oral administration, one capsule of each, twice per day during breakfast and dinner for 3 months. Patients in the treated group were provided with plastic bottles containing the exact number of capsules necessary to complete the treatment. The choice of using Lactobacillales and Bifidobacteria was driven by scientific evidence. In fact, several studies have demonstrated a healthy-orientated intestinal environment modulation by using probiotic products containing Bifidobacterium and Lactobacillus strains [42].

Patients randomized to receive the placebo were provided with the same amount of placebo capsules covering and miming the entire treatment cycle with probiotics. Placebo capsules were prepared ad hoc by a galenic local pharmacy to look exactly as probiotic capsules. Even placebo plastic containers looked exactly as those of probiotics. To assure that patients had not discontinued capsules assumption during the treatment period, and to check if any side effect had occurred, the nutritionist (T.M.) in our team had to phone each patient daily during the first week of treatment, and every week afterward. At the end of treatment cycle, both groups underwent a follow-up visit in which a re-evaluation of all parameters assessed at baseline, was performed. Any capsule not taken by the patients had to be returned and counted at the end-ofstudy visit to evaluate the compliance [43].

The primary outcome of ProbiotiCKD study was the urinary indican and 3-MI concentration after the treatment period.

The secondary outcomes were the after treatment concentrations of fecal Lactobacillales and Bifidobacteria and biochemistry laboratory parameters.

\section{Statistical analysis}

The data obtained have undergone to statistical analysis conducted with SPSS software (version 20.0) and PASS 11 (NCSS LLC., Kaysville, Utah). Continuous variables were reported as either mean \pm standard deviation (SD) or median and interquartile range (IQR) based on their distribution. Student's paired $t$ test or non-parametric Wilcoxon test, were used to examine the within-group differences between baseline and end-of-study visit, for normally distributed or skewed variables, respectively. Comparisons of betweengroups changes from baseline were assessed by means of simple $t$ test or Mann-Whitney $U$ test according to distribution. Pearson's correlation coefficient was determined for the relationship between baseline and post-treatment levels of urinary 3-MI and indican each other and with clinical, laboratory and microbiological parameters.

The level of significance was set at a $p$ value $<0.05$.

\section{Sample size calculation}

Sample size was calculated considering difference in urinary indican and 3-MI concentrations after treatment as primary outcome. A sample size of 14 patients per group achieve $80 \%$ power to detect a difference of 1.1 in term of effect size (large effect size) with a significance level (alpha) of 0.05 and using a two-sided independent sample $t$ test.

Sample size was calculated considering difference in urinary indican and 3-MI concentrations after treatment as primary outcome.

\section{Results}

The anthropometric, clinical and demographic features of 28 recruited patients are resumed in Table 1. Patients had heterogeneous underlying renal disease and overall pharmacological therapy was similar in the two groups. No patient had nephrotic proteinuria or hypoalbuminemia. No therapeutic change was installed during the follow-up period. Statistical comparisons of the placebo and treatment groups were not significantly different.

As for nutritionist evaluation of dietary diaries, no relevant differences were observed between groups. Moreover, baseline dietary standardized conditions were achieved (protein, potassium and fibers intake), according to protocol requirements.

Fecal Lactobacillales and Bifidobacteria concentrations were evaluated at baseline. In overall cohort, according to healthy status-associated ranges $[4,5]$, an insufficient fecal concentration of both Lactobacillales (mean $2.3 \times 10^{3} \mathrm{CFU} /$ gr) and Bifidobacteria $\left(1.7 \times 10^{4} \mathrm{CFU} / \mathrm{gr}\right)$ was observed in 92.8 and $95.7 \%$ of patients, respectively (Table 2 ). 
Table 1 Anthropometric and clinical characteristics at baseline

\begin{tabular}{|c|c|c|}
\hline Basal parameters & Placebo group $(n=14)$ & Probiotics group $(n=14)$ \\
\hline Gender $(\mathrm{M} / \mathrm{F})(n)$ & $6 / 8$ & $9 / 5$ \\
\hline Age (years) & $58.2 \pm 6.2$ & $61.3 \pm 5.2$ \\
\hline Weight (kg) & $74.6 \pm 3.8$ & $77.1 \pm 1.9$ \\
\hline BMI & $26.2 \pm 2.7$ & $25.2 \pm 3.1$ \\
\hline Systolic blood pressure (mmHg) & $130 \pm 14.7$ & $132 \pm 14.2$ \\
\hline Diastolic blood pressure (mmHg) & $82 \pm 6.7$ & $84 \pm 8.6$ \\
\hline Glucose (mg/dl) & $99.6 \pm 14.5$ & $98.9 \pm 12.6$ \\
\hline BUN (mg/dl) & $79.5 \pm 12.5$ & $80.5 \pm 10.6$ \\
\hline Serum creatinine $(\mathrm{mg} / \mathrm{dl})$ & $1.8 \pm 0.3$ & $1.78 \pm 0.4$ \\
\hline EPI-eGFR (ml/min/1.73 m²) & $48.4 \pm 7.4$ & $49.3 \pm 5.8$ \\
\hline Cystatin C (mg/dl) & $1.0 \pm 0.3$ & $0.99 \pm 0.1$ \\
\hline Uric acid (mg/dl) & $4.7 \pm 0.5$ & $4.6 \pm 0.4$ \\
\hline Albumin (g/dl) & $4.16 \pm 0.2$ & $4.05 \pm 0.1$ \\
\hline TSAT $(\%)$ & $18.4 \pm 6.8$ & $19.4 \pm 5.4$ \\
\hline Iron (mcg/dl) & $57.4 \pm 9.7$ & $60.7 \pm 8.7$ \\
\hline Transferrin (g/l) & $2.25 \pm 0.10$ & $2.40 \pm 0.10$ \\
\hline Ferritin (ng/ml) & $181 \pm 65.5$ & $177.9 \pm 152.3$ \\
\hline Hemoglobin (g/dl) & $12.3 \pm 2.6$ & $13.2 \pm 3.3$ \\
\hline Phosphate (mg/dl) & $3.2 \pm 0.2$ & $3.3 \pm 0.4$ \\
\hline Potassium (mmol/l) & $4.35 \pm 0.2$ & $4.29 \pm 0.1$ \\
\hline Calcium (mg/dl) & $8.8 \pm 0.2$ & $8.7 \pm 0.1$ \\
\hline Magnesium (mg/dl) & $2.03 \pm 0.10$ & $2.06 \pm 0.9$ \\
\hline Sodium $(\mathrm{mmol} / \mathrm{l})$ & $141.2 \pm 0.8$ & $142.1 \pm 0.9$ \\
\hline Total cholesterol (mg/dl) & $165 \pm 12.8$ & $158 \pm 14.3$ \\
\hline HDL cholesterol (mg/dl) & $44 \pm 7$ & $46 \pm 5$ \\
\hline Triglycerides (mg/dl) & $143 \pm 21$ & $152 \pm 14$ \\
\hline iPTH (pg/ml) & $203 \pm 70.8$ & $218.9 \pm 56.2$ \\
\hline C-reactive protein $(\mathrm{mg} / \mathrm{l})$ & $23.6 \pm 13.9$ & $25.5 \pm 15.4$ \\
\hline $\mathrm{B}_{2}$-microglobulin (mg/l) & $5.6 \pm 2.7$ & $6.4 \pm 1.8$ \\
\hline Blood bicarbonate (mmol/l) & $23.8 \pm 2.1$ & $24.1 \pm 1.7$ \\
\hline Estimated dietary protein intake (g/Kg/day) & $0.86 \pm 1.5$ & $0.88 \pm 1.3$ \\
\hline Estimated calories intake (cal/Kg/die) & $34 \pm 7$ & $33 \pm 5$ \\
\hline ACE inhibitor/AT1-receptor blockers $(n)$ & 12 & 14 \\
\hline Vit $\mathrm{D}$ analogues $(n)$ & 1 & 0 \\
\hline Furosemide/hydrochlorothiazide $(n)$ & 8 & 7 \\
\hline Oral antidiabetic drugs & 4 & 2 \\
\hline Therapy with insulin & 1 & 2 \\
\hline Eritropoietin & 0 & 0 \\
\hline Therapy with phosphate chelants & 0 & 0 \\
\hline Therapy with statins/fibrates & 11 & 13 \\
\hline \multicolumn{3}{|l|}{ Kidney diseases } \\
\hline Nephroangiosclerosis $(n)$ & 4 & 5 \\
\hline Non-nephrotic diabetic nephropathy $(n)$ & 5 & 4 \\
\hline Autosomal polycystic kidney disease $(n)$ & 2 & 1 \\
\hline Non-nephrotic unknown nephropathy $(n)$ & 2 & 2 \\
\hline Biopsy proven IgA nephropathy $(n)$ & 1 & 2 \\
\hline
\end{tabular}

At baseline, a direct highly significant correlation was found between urinary indican and 3-MI each other, and of both metabolites with serum levels of C-reactive protein
(CRP), ferritin and $\beta 2$-microglobulin. An inverse correlation was also discovered between urinary metabolites and fecal concentrations of Lactobacillales and Bifidobacteria, 
Table 2 The impact of ProbiotiCKD administration protocol on fecal Lactobacillales and Bifidobacteria concentrations

\begin{tabular}{|c|c|c|c|c|c|c|c|}
\hline \multirow{2}{*}{$\begin{array}{l}\text { Mean fecal bacteria } \\
\text { concentration }(\mathrm{CFU} / \mathrm{g})\end{array}$} & \multicolumn{2}{|l|}{ Placebo group } & \multirow[t]{2}{*}{$p$ value } & \multicolumn{2}{|l|}{ Probiotics group } & \multirow[t]{2}{*}{$p$ value } & \multirow{2}{*}{$\begin{array}{l}p \text { value placebo } \\
\text { (AT) vs probiotics } \\
\text { (AT) }\end{array}$} \\
\hline & Before treatment & After treatment & & Before treatment & After treatment & & \\
\hline Lactobacillales & $2.3 \times 10^{3}$ & $1.9 \times 10^{3}$ & ns & $2.1 \times 10^{3}$ & $2.2 \times 10^{6}$ & $<0.001$ & $<0.001$ \\
\hline Bifidobacteria & $1.7 \times 10^{4}$ & $1.8 \times 10^{4}$ & ns & $1.9 \times 10^{4}$ & $2.5 \times 10^{7}$ & $<0.001$ & $<0.001$ \\
\hline
\end{tabular}

Data are reported as mean \pm standard deviation. Statistical significance was set at a $p$ value $>0.05$

CFU/g colony-forming unit/g; $A T$ after treatment; $n s$ not significant

Table 3 Correlations between indican and 3-MI each other, and with clinical, laboratory and microbiological variables, at baseline

\begin{tabular}{|c|c|c|c|c|}
\hline \multirow[t]{2}{*}{ Parameters } & \multicolumn{2}{|l|}{ Indican } & \multicolumn{2}{|l|}{ 3-MI } \\
\hline & $r$ & $p$ value & $r$ & $p$ value \\
\hline Indican & - & - & 0.842 & $<0.001$ \\
\hline 3-MI & 0.842 & $<0.001$ & - & - \\
\hline CRP & 0.891 & $<0.001$ & 0.865 & $<0.001$ \\
\hline Ferritin & 0.523 & $<0.001$ & 0.529 & $<0.001$ \\
\hline eGFR & -0.611 & $<0.001$ & -0.600 & $<0.001$ \\
\hline $\begin{array}{l}\text { Fecal Lacto- } \\
\text { bacillales } \\
\text { concentrations }\end{array}$ & -0.842 & $<0.001$ & -0.792 & $<0.001$ \\
\hline $\begin{array}{l}\text { Fecal Bifidobac- } \\
\text { teria concen- } \\
\text { trations }\end{array}$ & -0.855 & $<0.001$ & -0.799 & $<0.001$ \\
\hline
\end{tabular}

as well as eGFR. No correlation with age, gender, blood pressure, and BMI was found. In Table 3 are reported all $\mathrm{r}$ and $p$ values.

Urinary indican and 3-MI levels were used in ProbiotiCKD study to evaluate the possible predominance of either a fermentative and/or a putrefactive metabolism of gut microbiota in our CKD patients [20-23]. Remarkably, at baseline, both groups exhibited high-indican urinary levels as expression of a microbiota disequilibrium in the small bowel, thus suggesting an excessive gut bacterial fermentative metabolism. In particular, at baseline, indican averaged $28.2 \pm 9.3 \mathrm{mg} / \mathrm{l}(31.6 \pm 7.2 \mathrm{mg} / \mathrm{g}$ after correction by creatininuria) in probiotics group and $25 \pm 8.2 \mathrm{mg} / \mathrm{l}$ $(35.7 \pm 6.8 \mathrm{mg} / \mathrm{g}$ after correction by creatininuria) in placebo group (Fig. 2).

A putrefactive component of gut microbiota metabolism in the colon was also evident in both groups at baseline, as mean urinary 3 -MI was $16.2 \pm 2.7 \mu \mathrm{g} / \mathrm{l}(23.1 \pm 1.8 \mu \mathrm{g} / \mathrm{g}$ after correction by creatininuria) in probiotics group and $15.8 \pm 3.8 \mu \mathrm{g} / \mathrm{l}(22.6 \pm 2.1 \mu \mathrm{g} / \mathrm{g}$ after correction by creatininuria) in the placebo group (Fig. 3).

Notably, gut microbiota-related variables significantly improved after treatment in the probiotics group, while no change was observed in the placebo group. In fact, mean fecal Lactobacillales and Bifidobacteria concentrations raised to $2.1 \times 10^{6} \mathrm{CFU} / \mathrm{g}(p<0.001)$ and $1.9 \times 10^{7} \mathrm{CFU} / \mathrm{g}$ $(p<0.001)$, respectively, only in the probiotics group. The changes from baseline in fecal Lactobacillales and Bifidobacteria concentrations were significantly different between the placebo and probiotic groups $(p<0.001)$ (Table 2).

In addition, probiotics-treated patients exhibited a significant reduction of both indican and 3-MI urinary levels occurred in $96.4 \%(n=27)$ and $89.2 \%(n=24)$ of cases, respectively (see Figs. 2, 3 for details). By contrast, no difference was observed in the placebo group. After treatment with probiotics, in fact, mean urinary indican significantly decreased to $7.1 \pm 5.2 \mathrm{mg} / \mathrm{l}(p<0.001)[9.8 \pm 4.3 \mathrm{mg} / \mathrm{l}$ after correction by creatininuria $(p<0.001)]$, while it remained significantly higher in the placebo group $(24.4 \pm 7.1 \mathrm{mg} / \mathrm{l}$, $p<0.001)$, even after correction by urine creatinine $(35.4 \pm 4.8 \mathrm{mg} / \mathrm{g}, p<0.001)$ (Fig. 2). Similarly, mean 3-MI urinary level was significantly decreased only in the probiotics group $[3.3 \pm 2.1 \mu \mathrm{g} / \mathrm{l}(p<0.001)]$ as confirmed also after correction by creatininuria $[4.7 \pm 3.2 \mu \mathrm{g} / \mathrm{g}(p<0.001)]$. Conversely, no change occurred $[14.7 \pm 2.7 \mu \mathrm{g} / \mathrm{l}(18.8 \pm 3.9 \mu \mathrm{g} / \mathrm{g}$ after correction by creatininuria)] in the control group after treatment. The urinary indican and 3-MI levels after the treatment period were significantly lower in the probiotics patients as compared to the placebo group (Fig. 3).

The impact of probiotics on several lab parameters is reported in Table 4. Of note, compared to baseline, mean serum iron level was significantly increased from $58.7 \pm 16.7 \mu \mathrm{g} / \mathrm{dl}$ at baseline to $66.7 \pm 14 \mu \mathrm{g} / \mathrm{dl}(p<0.001)$ after treatment only in the probiotics group. Accordingly, mean transferrin saturation (TSAT) was also increased in response to probiotics administration from $19.4 \pm 5.4 \%$ at baseline to $21.7 \pm 3.7 \%(p<0.001)$ after treatment. Conversely, a slight TSAT reduction was observed in the placebo group, in which mean baseline TSAT $18.4 \pm 6.8 \%$ decreased to $17 \pm 5.3 \%(p=0.048)$. In the probiotics group, basal mean ferritin decreased from $167.9 \pm 152.3$ to $141 \pm 113.6 \mathrm{ng} / \mathrm{ml}$ $(p<0.001)$ after the treatment cycle, while it remained stable in the placebo group.

Moreover, mean C-reactive protein (CRP) was significantly lower after the treatment with probiotics, changing from $25.5 \pm 15.4$ to $9.7 \pm 7.3 \mathrm{mg} / \mathrm{l}(p<0.001)$. No significant variation was instead observed in the placebo group. 


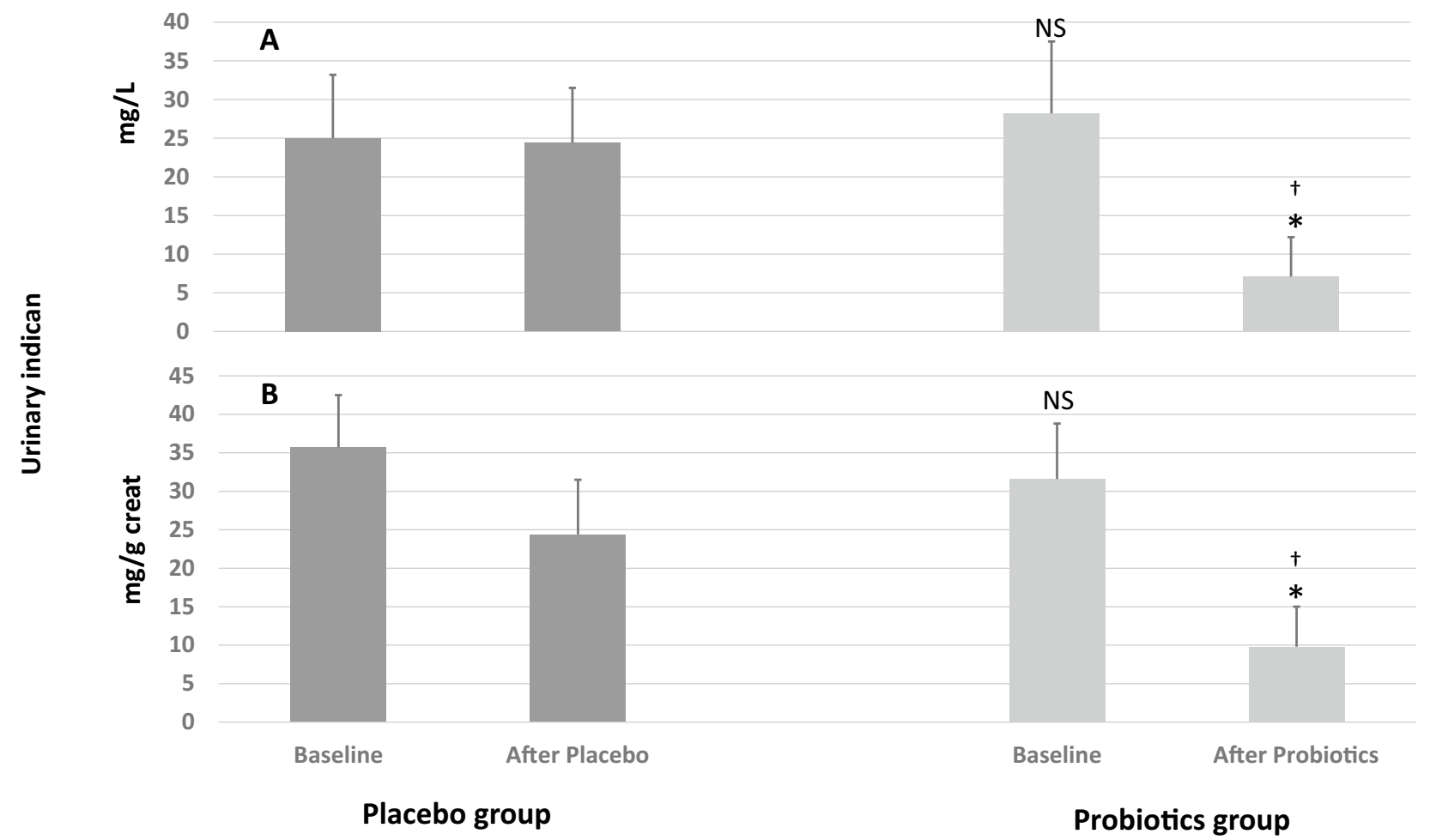

Fig. 2 The impact of ProbiotiCKD administration protocol on urinary indican in $\mathrm{mg} / \mathrm{l}$ (a) and and as corrected by urinary creatinine $(\mathrm{mg} / \mathrm{g}$ creatininuria) (b). Data are reported as mean \pm standard deviation.

Conversely to placebo, iPTH did not increase in the probiotics group, in which a significant increase in mean serum calcium was also observed (see Table 4 for details).

Even total cholesterol and triglycerides both significantly decreased only in the probiotics group, as reported in Table 4. However, basal mean values of these parameters were within normal range.

Notably, mean $\beta 2$-microglobulin levels showed an opposite trend in the two groups: it decreased in probiotics group from $6.4 \pm 1.8 \mathrm{mg} / \mathrm{l}$ at baseline to $4.1 \pm 1.3 \mathrm{mg} / \mathrm{l}(p<0.001)$ after the treatment cycle and increased in the placebo group from $5.6 \pm 2.7 \mathrm{mg} / \mathrm{l}$ at baseline to $7.5 \pm 2.7 \mathrm{mg} / \mathrm{l}(p<0.001)$ at follow-up visit. Conversely, eGFRat end-of-study visit was not different from baseline in both groups.

Compliance to treatment was overall good, as the mean number of capsules taken by patients in both groups was $438 \pm 4$ out of the 465 capsules provided to each patient for the entire treatment. Of note, none of the patients discontinued the treatment and no relevant side effects were reported.
Statistical significance was set at a $p$ value $>0.05$. NS not significant vs placebo baseline; $(*)$ vs basal $(p<0.001)$; $(\dagger)$ vs placebo after placebo $(p<0.001)$

\section{Discussion}

Gut dysbiosis is defined as an imbalanced intestinal microbial community with quantitative and qualitative alterations in the composition and in metabolic activities of the gut microbiota. The criteria for dysbiosis diagnosis include evaluation of bacterial microflora and its metabolism biomarkers. Dysbiosis is a disorder often associated with diverse diseases, such as hypertension, atherosclerosis, obesity, type 2 diabetes, inflammatory bowel and cardiovascular diseases [44]. Moreover, preliminary evidences indicate that the quantitative and qualitative profile of microbiota might be altered in patients with CKD, as reported in studies performed prevalently in patients with ESRD and on chronic hemodialysis [45]. The gut dysbiosis in uremia depends on several factors. In fact, urea secretion increases in the gastrointestinal district with kidney function loss, leading to more ammonia formation 


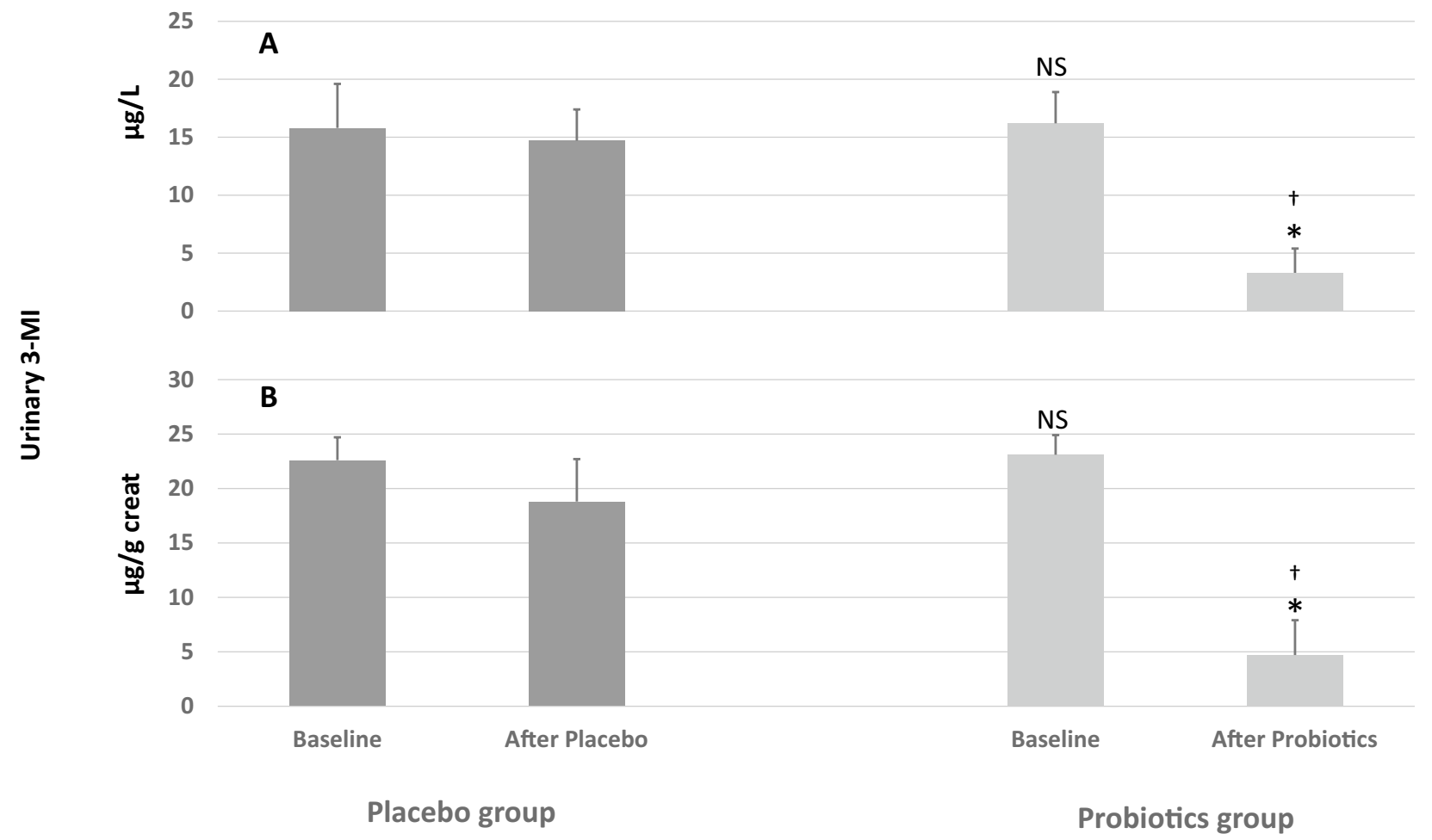

Fig. 3 The impact of ProbiotiCKD administration protocol on urinary 3- $\mathrm{MI}$ in $\mu \mathrm{g} / \mathrm{l}$ (a) and as corrected by urinary creatinine ( $\mu \mathrm{g} / \mathrm{g}$ creatininuria) (b). Data are reported as mean \pm standard deviation. Statisti- cal significance was set at a $p$ value $>0.05$. NS not significant vs placebo baseline; $(*)$ vs basal $(p<0.001) ;(\dagger)$ vs placebo after placebo $(p<0.001)$

Table 4 Most significant changes of lab parameters in response to ProbiotiCKD administration protocol

\begin{tabular}{|c|c|c|c|c|c|c|}
\hline \multirow[t]{2}{*}{ Lab parameters } & \multicolumn{3}{|c|}{ Placebo group $(n=14)$} & \multicolumn{3}{|c|}{ Probiotics group $(n=14)$} \\
\hline & Baseline & After placebo & $p$ value & Baseline & After treatment & $p$ value \\
\hline Iron $(\mathrm{mcg} / \mathrm{dl})$ & $58.8 \pm 7.6$ & $57.5 \pm 7.4$ & $n s$ & $58.7 \pm 16.7$ & $66.7 \pm 14$ & $<0.001$ \\
\hline TSAT (\%) & $18.4 \pm 6.8$ & $17 \pm 5.3$ & 0.048 & $19.4 \pm 5.4$ & $21.7 \pm 3.7$ & $<0.001$ \\
\hline Ferritin (ng/ml) & $165.5 \pm 115.3$ & $167.6 \pm 128.3$ & ns & $167.9 \pm 152.3$ & $141 \pm 113.6$ & $<0.001$ \\
\hline C-reactive protein $(\mathrm{mg} / \mathrm{l})$ & $25.8 \pm 8.9$ & $26.5 \pm 3.5$ & ns & $25.5 \pm 15.4$ & $9.7 \pm 7.3$ & $<0.001$ \\
\hline Total cholesterol $(\mathrm{mg} / \mathrm{dl})$ & $165 \pm 12.8$ & $162 \pm 13.3$ & ns & $158 \pm 14.3$ & $151 \pm 12.3$ & $<0.01$ \\
\hline HDL cholesterol $(\mathrm{mg} / \mathrm{dl})$ & $44 \pm 7$ & $45 \pm 5$ & ns & $46 \pm 5$ & $44 \pm 4$ & ns \\
\hline Triglycerides (mg/dl) & $143 \pm 21$ & $140 \pm 18$ & ns & $152 \pm 14$ & $140 \pm 12$ & $<0.01$ \\
\hline iPTH (pg/ml) & $203 \pm 70$ & $235 \pm 67$ & 0.03 & $218 \pm 56$ & $220 \pm 63$ & ns \\
\hline Calcium (mg/dl) & $8.8 \pm 0.2$ & $8.7 \pm 0.4$ & ns & $8.7 \pm 0.1$ & $9.0 \pm 0.3$ & 0.03 \\
\hline$\beta_{2}$-microglobulin (mg/l) & $5.6 \pm 2.7$ & $7.5 \pm 2.7$ & $<0.001$ & $6.4 \pm 1.8$ & $4.1 \pm 1.3$ & $<0.001$ \\
\hline eGFR (ml/min/1.73 m²) & $48.4 \pm 7.4$ & $48.6 \pm 7.1$ & 0.765 & $49.3 \pm 5.8$ & $49.0 \pm 5.0$ & 0.490 \\
\hline
\end{tabular}

Data are reported as mean \pm standard deviation. Statistical significance was set at a $p$ value $>0.05$

$n s$ not significant

that reduces commensal bacteria growth [46]. Decreased fibers dietary intake, slow colonic transit, metabolic acidosis, intestinal wall edema and possible oral iron intake might be additional pro-dysbiosis factors [47, 48]. Despite its relevance, gut dysbiosis is gaining the attention of the scientific community only in recent years and many aspects of this condition still remain open issues.

ProbiotiCKD is a single-center, prospective, randomized, placebo-controlled study designed to evaluate the intestinal microbiota and its modifications in response to a novel 
probiotics administration protocol in a population of CKD patients at stage 3a KDIGO. At this aim, fecal Lactobacillales and Bifidobacteria concentrations were determined and urinary indican and 3-MI levels were measured. The most surprising result observed in ProbiotiCKD is the evidence of an unhealthy gut microbiota even in patients with eGFR between 60 and $45 \mathrm{ml} / \mathrm{min} / 1.73 \mathrm{~m}^{2}$. The scarce representation of fecal Lactobacillales and Bifidobacteria combined with the high-urinary indican and 3-MI levels, both widely observed in our cohort, demonstrates that even such a residual renal function is insufficient to maintain a healthy balance in gut microbiota in early CKD. Therefore, in ProbiotiCKD study, we observed a significant alteration in both gut microbiota composition and intestinal bacterial metabolism in most of the patients. In support, Bifidobacteria have been shown to produce short-chain fatty acids, in particular butyrate, through a cross-feeding mechanism stimulating the growth of other bacterial species such as Lactobacillales. Butyrate stimulates the production of antimicrobial peptides (AMp), and the expression and activity of intestinal alkaline phosphatase (IAP) with an important role in the maintenance of intestinal homeostasis [49]. We guess that the low fecal concentration of both Bifidobacteria and Lactobacillales observed at baseline, may explain the altered gut microbiota metabolism observed in our patients. In support, we found an inverse correlation between urinary indican and both Lactobacillales and Bifidobacteria fecal concentrations. The supplementation of 'good' bacteria strains with Ramnoselle and Bifiselle, after having improved gut ambient with Enterelle, has possibly influenced the engraftment and growth of Bifidobacteria and Lactobacillales in the gut, with reduced production of gut dysbiosis metabolites. In fact, the coexistence of abnormally high-urinary levels of both 3-MI and indican in most of patients $(n=26)$ at baseline was significantly reduced after probiotics administration with consequent correction of dysbiosis in both putrefactive and fermentative components.

It is also important to emphasize that 3-MI and indican should not be considered just as type of dysbiosis biomarkers, but also as gut-derived uremic toxins. In particular, indican causes endothelial cell dysfunction and damage and is associated with tubulo-interstitial fibrosis, aortic calcification, vascular stiffness. Moreover, in patients with renal dysfunction, indican is the predictor of CKD progression and increases the overall and cardiovascular mortality risk [50]. Similarly, as observed in human and animal studies, also 3-MI induces glomerular sclerosis, interstitial fibrosis and is a predictor of mortality and cardiovascular events in patients with CKD [51]. Therefore, dysbiosis represents a signal from gut triggering inflammation and cardiovascular damage in CKD, since the initial stages of the disease. Accordingly to Ramezani et al., not only it is time for interventions aimed at blocking microbiota-related pathogenic biochemical pathways to ameliorate uremic syndrome [52], but also to anticipate the treatment to the early stage of renal insufficiency. This strategy could contribute to reduce the high mortality and comorbidity rate observed in the advanced stages of CKD.

ProbiotiCKD is the first intervention study performed in dysbiotic patients in early CKD stages, i.e., when GFR is greater than $44 \mathrm{ml} / \mathrm{min} / 1.73 \mathrm{~m}^{2}$. Actually, a previous, randomized trial tested the impact of probiotics administration on microbiota metabolites, but it was carried out in CKD patients with lower residual renal function (GFR $\leq 30 \mathrm{ml} /$ min) than in our cohort. In addition, in these patients, the treatment with probiotics resulted effective in reducing p-cresil-glucuronide levels, but ineffective in lowering indican levels [53]. In comparison to our study, a more advanced stage of renal insufficiency and the different type and modality of probiotics administration might have influenced the different results.

It is evident that an optimization of probiotics administration protocols is needed. Gut dysbiosis treatment implies the use of good quality probiotics. Probiotics are live and vital microorganisms able to benefit the host if consumed in an adequate amount, as part of a food or a supplement [54]. To be considered probiotics, microorganisms have to be normal components of human gut microbiota with effective delivery in the intestinal district of at least $10^{7}-10^{9}$ cells per day [55]. Consequently, probiotics should be resistant to sudden $\mathrm{pH}$ changes due to the exposure to gastric and pancreatic juice and bile. In some cases, different factors (unsuitable intestinal environment, insufficient dose, poor quality products) may interfere with probiotics effectiveness in correcting gut dysbiosis. The probiotics that we used in our study were high-quality products accomplishing to Italian Minister of Health criteria, and our novel ProbiotiCKD administration protocol was accurately projected. The rationale was based on creating a favorable intestinal environment prior to gut colonization with probiotics. Moreover, a good delivery of probiotics in the gut was assured using high doses of quality probiotics masked to the stomach acidity by a gastro-resistant film. ProbiotiCKD administration protocol revealed its effectiveness in correcting CKD-related intestinal dysbiosis. Undoubtedly, more studies comparing even other probiotics administration protocols in larger populations with comparable residual renal function, and with a longer follow-up, would be desirable.

Species of Lactobacillus and Bifidobacterium are most commonly used as probiotics, but the yeast Saccharomyces boulardii and some E. faecium are also used. A number of healthy effects are associated with usage of probiotics. In fact, their administration has been shown to stimulate the immune response, have an anti-inflammatory effect and restore gut dysbiosis [56]. Enterococcus species, in particular E. faecium, have been widely used over the last decade in 
the food industry as probiotics or as starter cultures because they produce bacteriocins [57]. In ProbiotiCKD protocol, a washout period with E. faecium was planned because Enterococcus species, in particular E. faecium, have been widely used over the last decade in the food industry as probiotics or as starter cultures to produce bacteriocins. These antimicrobial peptides are ribosomally synthesized and released in the extracellular ambient to fight competing bacterial species. Moreover, Enterococcus species are known to produce a range of enterocins, including enterocins $\mathrm{A}, \mathrm{B}, \mathrm{I}, \mathrm{L}$ and $\mathrm{P}$, which are active against pathogen bacteria such as Staphylococcus aureus, Listeria and Clostridium species [57]. For all these reasons, the use of E. faecium within our 'sequential' probiotics administration protocol, preliminary to gut colonization with Bifidobacteria and Lactobacillales strains, was addressed to create a favorable intestinal ambient to the engraftment of these eubiotic bacteria [58].

In our investigation, we observed intriguing effects of the tested 'sequential' probiotics administration on different lab parameters. First, a significant reduction of CRP levels was obtained only in the treated group. Considering that a chronic inflammation state is associated to CKD and can influence several long-term clinical outcomes (e.g., highcardiovascular risk, anemia, and immunodepression), we believe that our observations could be relevant if translated to clinical practice. Moreover, we suggest that the antiinflammatory effect of sequentially administered probiotics might be also considered in other patient typologies (e.g., patients with cardiac, oncologic, gastroenteric, infectious, and hepatic diseases) [59-61].

Of note, an improvement in serum iron, ferritin and TSAT limited to patients treated with probiotics was observed in our study. According to Tursi et al. [62], we hypothesize that this result might depend on both the ameliorated iron reabsorption in the gut and the reduction of inflammation. Surprisingly, no effects of probiotics were observed with respect to hemoglobin levels despite the improved iron status, but we suppose that this result could emerge in a longer observation study. We are currently running another study aimed to better characterize the interference of ProbiotiCKD protocol on specific biomarkers of both inflammation and erytropoietic activity.

As for serum lipids levels, after treatment, an improvement of total cholesterol and triglycerides was observed only in the probiotics group. However, even at baseline, lipid control was overall satisfactory in both groups, possibly due to the large employment of statins and fibrates, as reported in Table 1. Anyway, our observation confirms the results of several other studies in which the consumption of probiotics reduced the systemic cholesterol levels and caused a decrease in triglycerides as well. This result appears relevant because it suggests an additional pathway of cardiovascular risk reduction linked to probiotics use.
Focusing on another fundamental clinical feature of $\mathrm{CKD}$, such as mineral bone disease (MBD), despite the short follow-up, in probiotics group, we did not observe any significant change in iPTH plasma levels, while serum calcium levels significantly improved. By contrast, iPTH increased and serum calcium remained unchanged in the placebo group. We hypothesize that the ameliorated calcium reabsorption due to gut dysbiosis correction, could have helped to prevent iPTH increase in the probiotics group. This result appears of great interest in CKD patients. A confirmation in a larger population with early stage CKD and with a longer follow-up would be an important focus for future investigations and to develop new treatment strategies aimed to prevent cardiovascular and mineral bone disease.

Another rather interesting result observed in our study is the opposite trend of $\beta 2$-microglobulin in the two groups. Specifically, $\beta 2$-microglobulin was correlated to urinary 3-MI and indican at baseline and decreased in the treated group after probiotics administration, while it was increased in the placebo group. Even this result is of great interest. Doubtless, further studies are needed to confirm our data and also for understanding the link existing between intestinal dysbiosis and $\beta 2$-microglobulin in CKD patients.

\section{Conclusions}

ProbiotiCKD is the first intervention study demonstrating that an intestinal mixed (fermentative and putrefactive) dysbiosis is present even in the earlier stages of CKD and that it can be effectively corrected by high-quality probiotics novel mode of administration tested in the study. Moreover, the intestinal dysbiosis correction was associated with improved CRP, iron status, iPTH and $\beta 2$-microglobulin only in the treated group. Consequently, we suggest that (a) the probiotics administration protocol employed in ProbiotiCKD can represent a valid therapeutic tool for an effective intestinal dysbiosis correction; (b) probiotics administration has been associated with positive reflexes on several important lab parameters; (c) the probiotics therapy could help reduce inflammation in CKD, with possible beneficial effects on cardio-renal outcomes, particularly if the treatment is early started. The latter aspect would need a more extensive study in a larger population and with a longer follow-up.

Funding Probiotics were kindly provided by Bromatech Ltd, Viale Premuda, 46-20129 Milano.

\section{Compliance with ethical standards}

Conflict of interest The authors declare that they have no conflict of interest. 
Ethical approval All procedures were conducted in accordance with the ethical standards of the regional research committee and with the 1964 Helsinki Declaration and its later amendments or comparable ethical standards. An informed consent was also obtained from all individual participants included in the study.

Informed consent An informed consent was also obtained from all individual participants included in the study.

Open Access This article is distributed under the terms of the Creative Commons Attribution 4.0 International License (http://creativeco mmons.org/licenses/by/4.0/), which permits unrestricted use, distribution, and reproduction in any medium, provided you give appropriate credit to the original author(s) and the source, provide a link to the Creative Commons license, and indicate if changes were made.

\section{References}

1. Thursby E, Juge N (2017) Introduction to the human gut microbiota. Biochem J 474:1823-1836. https://doi.org/10.1042/BCJ20 160510

2. Rodríguez JM, Murphy K, Stanton C et al (2015) The composition of the gut microbiota throughout life, with an emphasis on early life. Microb Ecol Health Dis 26:26050. https://doi.org/10.3402/ mehd.v26.26050

3. Claesson MJ, Jeffery IB, Conde S et al (2012) Gut microbiota composition correlates with diet and health in the elderly. Nature 488:178-184. https://doi.org/10.1038/nature11319

4. Kiliç GB, Karahan AG (2010) Identification of lactic acid bacteria isolated from the fecal samples of healthy humans and patients with dyspepsia, and determination of their ph, bile, and antibiotic tolerance properties. J Mol Microbiol Biotechnol 18:220-229. https://doi.org/10.1159/000319597

5. Martinello F, Roman CF, Souza PA (2017) Effects of probiotic intake on intestinal bifidobacteria of celiac patients. Arq Gastroenterol 54:85-90. https://doi.org/10.1590/S0004-2803.20170 0000-07

6. Lau K, Srivatsav V, Rizwan A et al (2017) Bridging the gap between gut microbial dysbiosis and cardiovascular diselases. Nutrients 9:. https://doi.org/10.3390/nu9080859

7. Simeoni M, Armeni A, Summaria C et al (2017) Current evidence on the use of anti-RAAS agents in congenital or acquired solitary kidney. Ren Fail 39:660-670. https://doi.org/10.1080/08860 22X.2017.1361840

8. Simeoni M, Cerantonio A, Pastore I et al (2016) The correct renal function evaluation in patients with thyroid dysfunction. J Endocrinol Invest 39:495-507. https://doi.org/10.1007/s4061 8-015-0402-8

9. Simeoni M, Damiano S, Capolongo G et al (2017) Rare renal diseases can be used as tools to investigate common kidney disorders. Kidney Dis Basel Switz 3:43-49. https://doi.org/10.1159/00047 5841

10. D’Onofrio G, Simeoni M, Rizza P et al (2017) Quality of life, clinical outcome, personality and coping in chronic hemodialysis patients. Ren Fail 39:45-53. https://doi.org/10.1080/08860 22X.2016.1244077

11. Kim M-S, Hwang S-S, Park E-J, Bae J-W (2013) Strict vegetarian diet improves the risk factors associated with metabolic diseases by modulating gut microbiota and reducing intestinal inflammation. Environ Microbiol Rep 5:765-775. https://doi. org/10.1111/1758-2229.12079
12. Vaziri ND, Wong J, Pahl M et al (2013) Chronic kidney disease alters intestinal microbial flora. Kidney Int 83:308-315. https:// doi.org/10.1038/ki.2012.345

13. Evenepoel P, Meijers BK (2012) Dietary fiber and protein: nutritional therapy in chronic kidney disease and beyond. Kidney Int 81:227-229. https://doi.org/10.1038/ki.2011.394

14. Mafra D, Barros AF, Fouque D (2013) Dietary protein metabolism by gut microbiota and its consequences for chronic kidney disease patients. Future Microbiol 8:1317-1323. https://doi. org/10.2217/fmb.13.103

15. Pham P-CT, Toscano E, Pham P-MT et al (2009) Pain management in patients with chronic kidney disease. NDT Plus 2:111-118. https://doi.org/10.1093/ndtplus/sfp001

16. Vitetta L, Gobe G (2013) Uremia and chronic kidney disease: the role of the gut microflora and therapies with pro- and prebiotics. Mol Nutr Food Res 57:824-832. https://doi.org/10.1002/ mnfr.201200714

17. Vaziri ND (2012) CKD impairs barrier function and alters microbial flora of the intestine: a major link to inflammation and uremic toxicity. Curr Opin Nephrol Hypertens 21:587-592. https://doi.org/10.1097/MNH.0b013e328358c8d5

18. Yokoyama MT, Carlson JR (1979) Microbial metabolites of tryptophan in the intestinal tract with special reference to skatole. Am J Clin Nutr 32:173-178. https://doi.org/10.1093/ ajcn/32.1.173

19. Bryan GT (1966) Quantitative studies on the urinary excretion of indoxyl sulfate (indican) in man following administration of L-tryptophan and acetyl-L-tryptophan. Am J Clin Nutr 19:105112. https://doi.org/10.1093/ajen/19.2.113

20. Hawrelak JA, Myers SP (2004) The causes of intestinal dysbiosis: a review. Altern Med Rev J Clin Ther 9:180-197 (PMID:15253677)

21. Richard S, Lord, Alexander Bralley J (2008) Clinical applications of urinary organic acids. Part 2. Dysbiosis markers. Altern Med Rev 13(4):292-306

22. Adike A, Di Baise JK (2018) Small intestinal bacterial overgrowth: nutritional implications, diagnosis, and management. Gastroenterol Clin North Am 47(1):193-208. https://doi. org/10.1016/j.gtc.2017.09.008

23. Cassani E, Barichella M, Cancello R et al (2015) Increased urinary indoxyl sulfate (indican): new insights into gut dysbiosis in Parkinson's disease. Parkinsonism Relat Disord 21(4):389-393. https://doi.org/10.1016/j.parkreldis.2015.02.004

24. Alou MT, Lagier J-C, Raoult D (2016) Diet influence on the gut microbiota and dysbiosis related to nutritional disorders. Human Microbiome J 9(1):3-11. https://doi.org/10.1681/ASN.20151 11285

25. Conlon MA, Bird AR (2015) The impact of diet and lifestyle on gut microbiota and human health. Nutrients 7(1):17-44. https:// doi.org/10.3390/nu7010017)

26. Schulman G (2012) A nexus of progression of chronic kidney disease: tryptophan, profibrotic cytokines, and charcoal. J Ren Nutr Off J Counc Ren Nutr Natl Kidney Found 22:107-113. https ://doi.org/10.1053/j.jrn.2011.10.035

27. Andersen K, Kesper MS, Marschner JA et al (2017) Intestinal dysbiosis, barrier dysfunction, and bacterial translocation account for CKD-related systemic inflammation. J Am Soc Nephrol JASN 28:76-83. https://doi.org/10.1681/ASN.2015111285

28. Cianfrone P, Simeoni M, Comi N et al (2017) How to improve duration and efficiency of the antiproteinuric response to Ramipril: RamiPROT-a prospective cohort study. J Nephrol 30:95-102. https://doi.org/10.1007/s40620-015-0256-3

29. Rossi M, Johnson DW, Morrison M et al (2016) Synbiotics easing renal failure by improving gut microbiology (SYNERGY): a randomized trial. Clin J Am Soc Nephrol CJASN 11:223-231. https://doi.org/10.2215/CJN.05240515 
30. Satsangi J, Silverberg MS, Vermeire S, Colombel JF (2006) The Montreal classification of inflammatory bowel disease: controversies, consensus, and implications. Gut 55(6):749-753. https://doi. org/10.1136/gut.2005.082909

31. Fuiano G, Zoccali C (2003) Guideline of conservative therapy of CKD. G Ita Nefrol 20(S-24):S48-S60

32. Agapito G, Simeoni M, Calabrese B et al (2018) DIETOS: a dietary recommender system for chronic diseases monitoring and management. Comput Methods Programs Biomed. 153:93-104. https://doi.org/10.1016/j.cmpb.2017.10.014 (Epub 2017 Oct 12)

33. Maroni BJ, Steinman TI, Mitch WE (1985) A method for estimating nitrogen intake of patients with chronic renal failure. Kidney Int 27:58-65 (PMID:3981873)

34. Brunius C, Vidanarachchi J, Tomankova J, Lundström K, Andersson K, Zamaratskaia G (2016) Skatole metabolites in urine as a biological marker of pigs with enhanced hepatic metabolism. Animal 10(10):1734-1740. https://doi.org/10.1017/S175173111 6000574

35. Laurino C, Palmieri B, Esposito A et al (2016) Effectiveness of a probiotic and prebiotic nutraceutical associated with controlled diet and hydrocolontherapy in the treatment of symptomatic intestinal dismicrobism. Min Gastrenterol Dietol 62(Suppl 2):1-19.

36. Greenberger N, Saegh S, Ruppert R (1968) Urine indican excretion in malabsorption disorders. Gastroenterol 55:204-211 (PMID: 4385691)

37. Patney NL, Mehrotra MP, Khanna HK, Kumar A (1976) Urinary Indican excretion in cirrhosis of liver. J Assoc Phys India 24(5):291-295 (PMID: 1022761)

38. Patney NL, Saxena SK, Mehrotra MP et al (1977) Urinary indican in diabetes mellitus. J Indian Med Assoc 68(5):94-97 (PMID: 903638)

39. Darkoh C, Chappell C, Gonzales C, Okhuysen P (2015) A rapid and specific method for the detection of indole in complex biological samples. Appl Environ Microbiol 81:8093-8097. https://doi. org/10.1128/AEM.02787-15

40. Gibson GR, Hutkins R, Sanders ME et al (2017) Expert consensus document: The International Scientific Association for Probiotics and Prebiotics (ISAPP) consensus statement on the definition and scope of prebiotics. Nat Rev Gastroenterol Hepatol 14:491-502. https://doi.org/10.1038/nrgastro.2017.75

41. Ramezani A, Raj DS (2014) The gut microbiome, kidney disease, and targeted interventions. J Am Soc Nephrol JASN 25:657-670. https://doi.org/10.1681/ASN.2013080905

42. Alatriste PVM, Arronte RU, Espinosa COG et al (2014) Effect of probiotics on human blood urea levels in patients with chronic renal failure. Nutr Hosp 29:582-590. https://doi.org/10.3305/ nh.2014.29.3.7179

43. Lee JK, Grace AK, Foster TG et al (2007) How should we measure medication adherence in clinical trials and practice? Ther Clin Risk Manag 3(4):685-690 (PMID: 18472991)

44. Sanders ME (2008) Probiotics: definition, sources, selection, and uses. Clin Infect Dis Off Publ Infect Dis Soc Am 46(Suppl 2):S58-S61. https://doi.org/10.1086/523341

45. Kang JY (1993) The gastrointestinal tract in uremia. Dig Dis Sci 38:257-268 (PMID:8425438)

46. Barreto FC, Barreto DV, Liabeuf S et al (2009) Serum indoxyl sulfate is associated with vascular disease and mortality in chronic kidney disease patients. Clin J Am Soc Nephrol CJASN 4:15511558. https://doi.org/10.2215/CJN.03980609

47. Ramezani A, Massy ZA, Meijers B et al (2016) Role of the gut microbiome in uremia: a potential therapeutic target. Am J
Kidney Dis Off J Natl Kidney Found 67:483-498. https://doi. org/10.1053/j.ajkd.2015.09.027

48. Sabatino A, Regolisti G, Brusasco I et al (2015) Alterations of intestinal barrier and microbiota in chronic kidney disease. Nephrol Dial Transplant Off Publ Eur Dial Transpl Assoc. Eur Ren Assoc 30:924-933. https://doi.org/10.1093/ndt/gfu287

49. Leccioli V, Oliveri M, Romeo M et al (2017) A new proposal for the pathogenic mechanism of non-coeliac/non-allergic gluten/ wheat sensitivity: piecing together the puzzle of recent scientific evidence. Nutrients. https://doi.org/10.3390/nu9111203

50. Park M, Hsu C-Y, Go AS et al (2017) Urine kidney injury biomarkers and risks of cardiovascular disease events and all-cause death: the CRIC study. Clin J Am Soc Nephrol CJASN 12:761771. https://doi.org/10.2215/CJN.08560816

51. Peyster E, Chen J, Feldman HI et al (2017) Inflammation and arterial stiffness in chronic kidney disease: findings from the CRIC study. Am J Hypertens 30:400-408. https://doi.org/10.1093/ajh/ hpw 164

52. Rampino T, Gregorini M, Camussi G et al (2005) Hepatocyte growth factor and its receptor Met are induced in crescentic glomerulonephritis. Nephrol Dial Transplant Off Publ Eur Dial Transpl Assoc Eur Ren Assoc 20:1066-1074. https://doi. org/10.1093/ndt/gfh740

53. Castello M, Gregorini M, Rampino T et al (2013) A retrospective analysis of dermatological lesions in kidney transplant patients. Indian J Med Res 137:1188-1192 (PMID:23852300)

54. Hill C, Guarner F, Reid G et al (2014) Expert consensus document. The International Scientific Association for Probiotics and Prebiotics consensus statement on the scope and appropriate use of the term probiotic. Nat Rev Gastroenterol Hepatol Aug;11(8):506-514. https://doi.org/10.1038/nrgastro.2014.66

55. Chen F, Jiang J, Tian D-D et al (2017) Targeting obesity for the prevention of chronic cardiovascular disease through gut microbiota-herb interactions: an opportunity for traditional herbs. Curr Pharm Des 23:1142-1152. https://doi.org/10.2174/1381612822 666161014115724

56. Gareau MG, Sherman PM, Walker WA (2010) Probiotics and the gut microbiota in intestinal health and disease. Nat Rev Gastroenterol Hepatol 7:503-514. https://doi.org/10.1038/nrgas tro.2010.117

57. Foulquie Moreno MR, Sarantinopoulos P, Tsakalidou E, De Vuyst L (2006) The role and application of Enterococci in food and health. Int J Food Microbiol 106:1-24. https://doi.org/10.1016/j. ijfoodmicro.2005.06.026

58. Campos CA, Rodriguez O, Calo-Mata P et al (2006) Preliminary characterization of bacteriocins from Lactococcus lactis, Enterococcus faecium and Enterococcus mundtii strains isolated from turbot (Psetta maxima). Food Res Int 39:356-364

59. Gao R, Gao Z, Huang L, Qin H (2017) Gut microbiota and colorectal cancer. Eur J Clin Microbiol Infect Dis Off Publ Eur Soc Clin Microbiol 36:757-769. https://doi.org/10.1007/s1009 6-016-2881-8

60. Lin L, Zhang J (2017) Role of intestinal microbiota and metabolites on gut homeostasis and human diseases. BMC Immunol 18:2. https://doi.org/10.1186/s12865-016-0187-3 (PMID:15973121)

61. Tang WHW, Hazen SL (2014) The contributory role of gut microbiota in cardiovascular disease. J Clin Invest 124:4204-4211. https ://doi.org/10.1172/JCI72331

62. Tursi A, Giorgetti GM, Brandimarte G, Elisei W (2005) High prevalence of celiac disease among patients affected by Crohn's disease. Inflamm Bowel Dis 11:662-666 